\title{
The effect of external ultrasound waves on ecchymosis, edema and pain after facial aesthetic surgery
}

\author{
Mohammed Taher Ahmed Omar, ${ }^{a}$ PTD; \\ Khaled EG, ${ }^{c}$ MD; Ahmed A Hassan, ${ }^{c}$ MD; Roqua Sabagh Mostafa, ${ }^{d}$ MD
}

a) Department of Physical Therapy for surgery, Faculty of Physical Therapy, Cairo, Egypt.

b) Member of Rehabilitation Research Chair, College of Applied Medical Science, King Saud University.

c) Department of Plastic Surgery, Ain Shams University, Cairo, Egypt.

d) Department of Physical Therapy Department, El-Matria Teaching Hospital, Cairo, Egypt.

\section{Corresponding Author: Mohammed Taher Ahmed Omar \\ Email address: Dr.Taher_M@Yahoo.Com - Momarar@ksu.edu.Sa \\ Mobile phone +20101547585 - +960542115404 \\ Faculty of Physical Therapy, Cairo University; \\ 7 Ahmad Alzayat St., Bain Elsarayat, Giza, Egypt.}

\begin{abstract}
Background and objective: Facial cosmetic procedures are often associated with unsightly postoperative ecchymosis, and swelling, which may be a source of pain and anxiety for patients and can be difficult to camouflage. This study was conducted to assess the safety and efficacy of low frequency $3 \mathrm{MHz}$ pulsed ultrasound (US) in the treatment of ecchymosis and swelling after facial cosmetic procedures.

Methods: Thirty patients undergoing facial cosmetic surgery had been involved and randomly divided into two equal groups. Ultrasound group (US-group, $n=15$ ), who received $3 \mathrm{MHz}$ pulsed US at $1.5 \mathrm{~W} / \mathrm{cm} 2$ for 5 minutes to each side of the face, and control group $(n=15)$. Postoperative assessment included severity of ecchymosis, postoperative pain, and amount of facial swelling. All measurements were performed by blinded investigator at postoperative days (PODs). ${ }^{1,3,5,7,10}$

Results: Complete resolution of ecchymosis, pain and facial swelling was observed between POD5 to POD7 in US group. While in control group resolution and reduction of pain and facial swelling occurred at POD7 to POD10.

Conclusion: Postoperative US therapy helps in early resolution and reduction of facial swelling and pain after facial cosmetic surgery with no reported complications or side effects.

Key words: Ultrasound, facial cosmetic surgery, ecchymosis, edema, pain.
\end{abstract}

\section{Introduction:}

Facial aesthetic surgeries are often associated with postoperative ecchymosis and edema. These may be a source of pain and anxiety for patients and can be difficult to camouflage. Ecchymosed and edematous tissue may require 10 days or more to resolve and potentially can limit social activities. ${ }^{1,2}$ 
Postoperative edema occurs due to changes in the capillary hemodynamic, loss of vascular integrity and lymphatic obstruction that collectively lead to accumulation of fluid in the tissue and result in visible swelling of the face. ${ }^{2}$

Ultrasound (US) has been used as a therapeutic modality for several decades, mainly by physiotherapists to treat soft-tissue injuries. ${ }^{3,4}$ Claims have been made that US modifies both the acute and chronic phases of inflammation. This led to reduction of edema, relieves pain, encourages healing, and modifies scar formation. ${ }^{5-7}$

The US is capable of producing therapeutic thermal and non-thermal effects in the tissues. $6,8,9$ The thermal effects may include increased blood flow, reduction of muscle spasm, increased tissue extensibility, and a mild inflammatory response. It is estimated that the thermal effects occur with elevation of tissue temperature to be $40-45^{\circ} \mathrm{c}$ for at least 5 minutes. ${ }^{31}$

It has been suggested that the non-thermal effects, including cavitations and acoustic microstreaming, are more important in the treatment of soft-tissue lesions than are the thermal effects. ${ }^{32}$ These may cause stimulation of fibroblast activity, increased protein synthesis, increased blood flow, and tissue regeneration. ${ }^{9}$

Despite the frequent use of US with reported success to clinical practice, ${ }^{8-12}$ its effect following facial aesthetic surgery has not been reported in the literature. Therefore, this study was designed to study and assess the efficacy of $3 \mathrm{MHz}$ pulsed mode US therapy on ecchymosis, edema, and pain after facial aesthetic surgeries.

\section{Patients and methods:}

The study included 30 patients who underwent surgical aesthetic procedures to the face from June 2008 to December 2010. The age of the patients ranged from 21 to 52 years old with an average age of 39 years. Patients who have had hypertension, diabetes, and abnormal bleeding and/or clotting profiles were excluded from the study. All patients were instructed to stop taking aspirin, non-steroidal anti-inflammatory drugs, vitamin E, and smoking for 2-week prior surgery. All surgical procedures were performed by consultant author. The surgical procedures included open rhinoplasty, facial contouring with fat injection, and blepharoplasty. No intraoperative homeostatic agents or drain were used. The patients were randomly assigned to ultrasound group $(n o=15)$ or control group (no=15) using enveloped methods.

\section{Ultrasound exposure protocol:}

The US therapy was conducted using (US700 ITO CO., LTD-Tokyo-Japan) 3MHz, with duty cycle of $20 \%$ and intensity of 0.5 $\mathrm{W} / \mathrm{cm}^{2}$. The transducer head had an area of $5.2 \mathrm{~cm}^{2}$, with an effective radiating area of $5 \mathrm{~cm}^{2}$. Sterilized US coupling media was applied to the skin. Then the US transducer head was placed firmly on the targeted skin surface, and pressed uniformly with rhythmical circular movement for lowering the power of the US transmission to maintain constant responses. ${ }^{2}$ After US application, gel was wiped off. Patients were instructed that if they encountered any other effects, they should contact the therapist promptly. The US therapy was administered daily, for 7 minutes on each side of the face starting at POD3 until the POD10 or until the signs, and symptoms resolved.

\section{Postoperative care:}

In all patients, standard pressure dressings were applied at the end of the procedure for 48 hours. Cryotherapy was applied to the surgical sites for the first 48 hours, which is routine in our practice. Cryotherapy was applied using 9-inch rounded-shaped ice bags and cold gel packs. The ice bag was applied to the surgical wound with the patient in the supine position with a head elevation of 30 degrees. At the same time, glasses-shaped cold gel packs were attached to the periorbital area with Velcro tape. Cryotherapy was applied for 20 minutes per hour, beginning three hours postoperatively, every hour except from 10pm-10am. Cryotherapy was applied by patients or caregivers after education about 
the protocol. Follow-up was made three times per day to confirm adequate application of the intervention.

The physician supplied the medications to ensure compliance. Diclofenac K (Novartis, Switzerland) was given $50 \mathrm{mg}, 3$ times daily for five days. All patients were placed on a fiveday antibiotic regimen $(500 \mathrm{mg}$ ampicillincloxacillin, Smith- Kline Beecham, England; 4 times daily). All the medications were administered orally.

\section{Clinical assessment:}

All patients were seen for assessment preoperatively and on PODs. $1,3,5,7,10$ Standard photographs were taken before and after treatment for the presence and changes of ecchymosis and edema.

Ecchymosis; each patient was digitally photographed on PODs. ${ }^{1,3,5,7,10}$ Three blinded surgeons evaluated the photographs and graded the ecchymosis on a scale of 0 to 3 , where; 0 , no ecchymosis, 1 , minimal ecchymosis, 2, moderate ecchymosis, 3, severe ecchymosis. Average mean scores were computed for all three observers. The statistical analysis was performed on the difference between the means scores. ${ }^{1}$

\section{Pain assessment:}

Patients were instructed to quantify their postoperative pain level in the morning of 10 consecutive days using visual analogue scale (VAS) prior to taking any pain medication. The VAS consisted of $10 \mathrm{~cm}$ line anchored at one end by the label 'No pain' and at the other end by 'Worst possible pain'. The patient marked on the line spot for the pain intensity which was then measured. ${ }^{10}$ Pain intensity was categorized into 0 as no pain, $1-3$ as mild pain, 4-6 as moderate pain, 7-9 as severe pain and 10 as worst pain.

\section{Facial edema assessment:}

As no published method satisfies all criteria for assessing facial swelling, we decided to use a measuring tape to measure facial width and swelling. The reference points distance used were the; ${ }^{11,12}$

1-Tragus - mental protuberance.
2-Tragus- mouth angle (corner of mouth).

3-Mandibular angle-nasal alae.

4-Mandibular angle - external eye angle.

5-Mandibular angle - internal eye angle.

6-Mentalprotuberance- external eye angle.

7-Mandibular angle-mental protuberance.

A single blinded therapist evaluated the amount of swelling by distance measurements performed between these well defined anatomical landmarks. The procedure was repeated three times on each patient, and the average was then taken (in $\mathrm{cm}$ ) and recorded. The extent of facial swelling was calculated through the sum of the following seven distances on each side of face and divided by two. The measurements were carried out just before the surgery and at PODs. $1,3,5,7,10$ Postoperative swelling was expressed as a percentage increase in facial width as follows: Postoperative measurement - Preoperative
measurement X100

Preoperative measurement

\section{Data analysis:}

Data were expressed as means \pm standard deviation (SD) unless otherwise indicated. Statistical differences between the two groups were tested with the unpaired t-test. Oneway analysis of variance used to determine significance within the group. The ecchymosis scores were compared using a Mann-Whitney test between the groups, and Wilcoxon test within the groups. The statistical package of social science software (SPSS Inc., Chicago, IL, USA) was used to perform the analysis. All $p$ values less than 0.05 were considered to be statistically significant.

\section{Results:}

Table(1) shows the clinical and operative characteristics of the patients who completed the study. The data regarding to age, sex, duration and types of surgeries were comparable between groups. A subgroup of patients undergoing facial aesthetic surgeries was created. They were identical in sex and age distribution, numbers and types of surgical procedures. Postoperative 
pain medication and antibiotic consumed by each patient within, and between groups was similar.

\section{Ecchymosis:}

Figure(1) shows observer's ecchymosis ratings scores according to days. At POD1, severe ecchymosis was reported in $(66.7 \%$ versus 60\%) and moderate ecchymosis in (33.3\% versus $40 \%)$ in US group versus control group. This revealed non significant differences $(\mathrm{P}>0.05)$ between the groups.

In the US group, substantial improvement and resolution of ecchymosis was observed between POD5 $(1.83 \pm 0.15, \mathrm{p}<0.05)$, POD7 $(0.83 \pm 1.33, \quad \mathrm{p}<0.05)$ compared to POD1 $(2.88 \pm 0.85)$ and POD3 (2.6 \pm 0.15$)$. Mean ecchymosis scores significantly declined to $(0.14 \pm 0.15, \mathrm{p}<0.05)$, at POD10, with a $95 \%$ improvement.

For control group, improvement and resolution of ecchymosis occurred between POD7 $(1.96 \pm 0.69, \quad \mathrm{p}<0.05)$ to POD10 $(0.91 \pm 0.45, \quad \mathrm{p}<0.05)$. There was no improvement in ecchymosis at POD5 $(2.33 \pm 0.45, \quad \mathrm{p}>0.05)$ compared to POD1 $(2.85 \pm 0.69)$ and POD3 $(2.57 \pm 0.69)$. The mean ecchymotic score improved by $86 \%$ at POD10. There was a significant reduction of ecchymosis between the US and control groups on PODs $5(\mathrm{p}<0.02), 7(\mathrm{p}<0.02)$, and $10(<0.01)$. In the US group, complete resolution of ecchymosis was observed in all patients at POD10. In the control group, ecchymosis resolved completely in 8 patients $(53.3 \%)$. Moderate ecchymosis has been reported in 3 patients (20\%) and 4 patients $(26.7 \%)$ had mild ecchymosis.

\section{Facial Edema:}

Figure(2), represents the mean percentage reduction of facial swelling in both groups. There was no significant difference in the average amount of swelling at POD1 $(20.7 \pm 2.3 \quad$ versus $19.27 \pm 3.2, \quad \mathrm{p}>0.05)$ and POD3 $(20.34 \pm 2.3$ versus $21.18 \pm 3.4$, $\mathrm{p}>0.05)$ between US and control groups respectively. Treatment with the US resulted in a continuous reduction of percentage of swelling between POD5 to POD10. There was a statistically significant reduction at POD5 (13.93 $\pm 4.5, \mathrm{p}=0.007)$, POD7 (8 \pm 3.6 , $\mathrm{p}=0.001)$, and POD10 $(3.11 \pm 1.88, \mathrm{p}=0.001)$ as compared to POD1 $(20.7 \pm 5.86)$ and POD3 (20.34 \pm 4.06$)$. For control group, the swelling was statistically significant at POD7 (11.64 $\pm 2.45, \mathrm{p}=0.03)$ and POD10 $(7.64 \pm 2.48, \mathrm{p}=0.01)$, while there was no improvement at POD5 $(18.22 \pm 4.45, \mathrm{p}>0.05)$ compared to POD1 $(19.27 \pm 5.69)$ and POD3 $(21.18 \pm 4.69)$. The percentage of the facial swelling revealed a significant reduction at POD5 (32.7\% versus 5.94\%, $\mathrm{p}=0.01)$, POD7 (61.35\% versus $39.59 \%, \mathrm{p}=0.001)$, POD10 $(84.97 \%$ versus $66.35 \%, \mathrm{p}=0.001)$ for US group versus control respectively.

\section{Postoperative pain:}

Figure (3) represents the mean pain scores. There were no significant differences in average pain intensity at POD1 $(6.73 \pm 1.53$ versus $6.46 \pm 1.5, \mathrm{p}=63)$ and POD3 $(3.13 \pm 1.53$ versus $4.48 \pm 1.5, \mathrm{p}=63$ ) between the US and control groups respectively. Chi-square test revealed non statistical significant difference $(\mathrm{P}>0.05)$ in the number of patients reporting severe pain $(8 ; 53.3 \%$ versus $7 ; 46.7 \%)$, moderate pain $(7 ; 46.7 \%$ versus $8 ; 53.3 \%)$ in US and control groups respectively. Treatment with US resulted in a significant reduction of postoperative pain between POD5 to POD7. Pain subsided completely in (9 patients; 60\%) by POD5, the remaining 6 patients had mild pain (3patients; 20\%) to moderate pain (3patient; 20\%). On POD7, 11 patients $(73.3 \%)$ reported no pain and 4 patients $(26.7 \%)$ had mild pain. At POD10, 13 patients $(86.7 \%)$ reported no pain and 2 patients $(13.3 \%)$ had mild pain. For control group, a significant reduction of postoperative pain was observed between POD5 to POD10. Pain subsided completely in 3 patients (20\%) by POD5. The remaining 12 patients had mild pain (3 patients; 20\%) to moderate pain (9 patients; 60\%). On POD7, 4 patients (26.7\%) reported no pain, and the 9 patients (60\%) had mild pain and 2 patients (13.3\%) had moderate pain. At POD10, 6 patients (40\%) reported no pain, 7 patients $(46.7 \%)$ had mild pain and 2 patients $(13.3 \%)$ had 


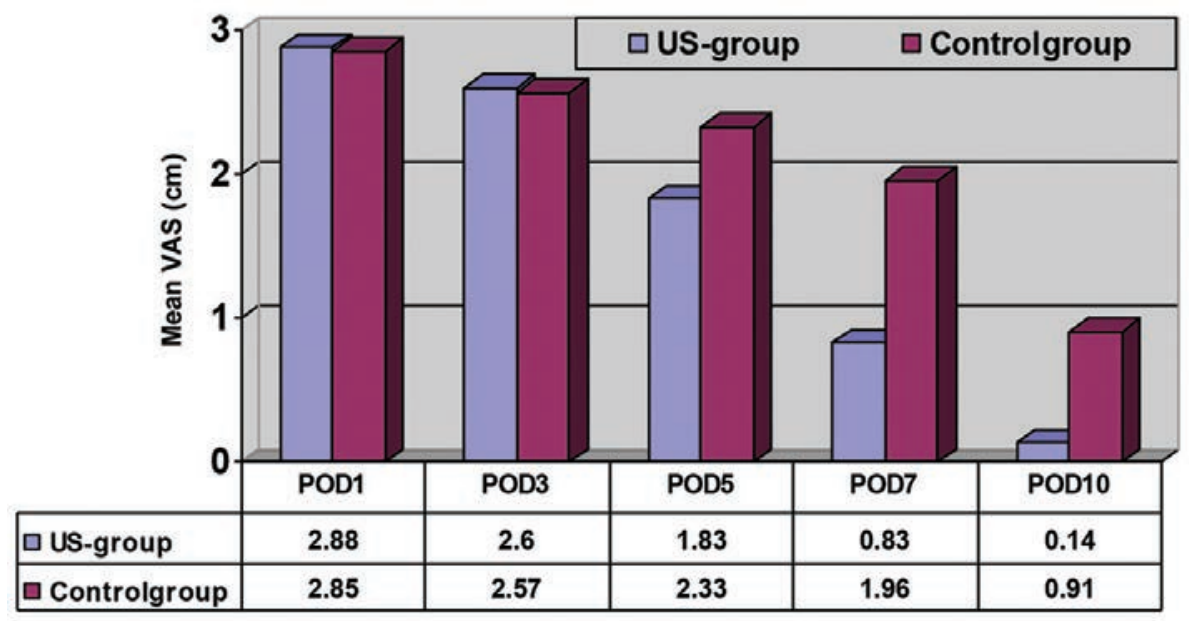

Figure (1): Observer's ecchymosis ratings scores.

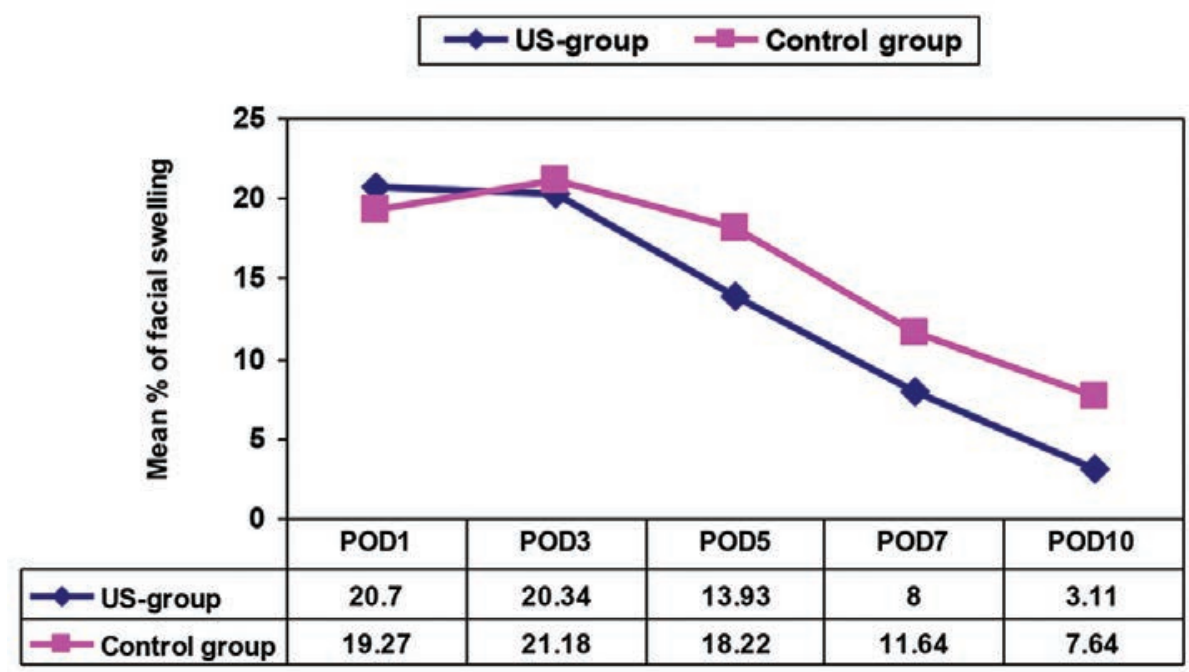

Figure (2): Progress of facial edema. POD: postoperative day. US: ultrasound.

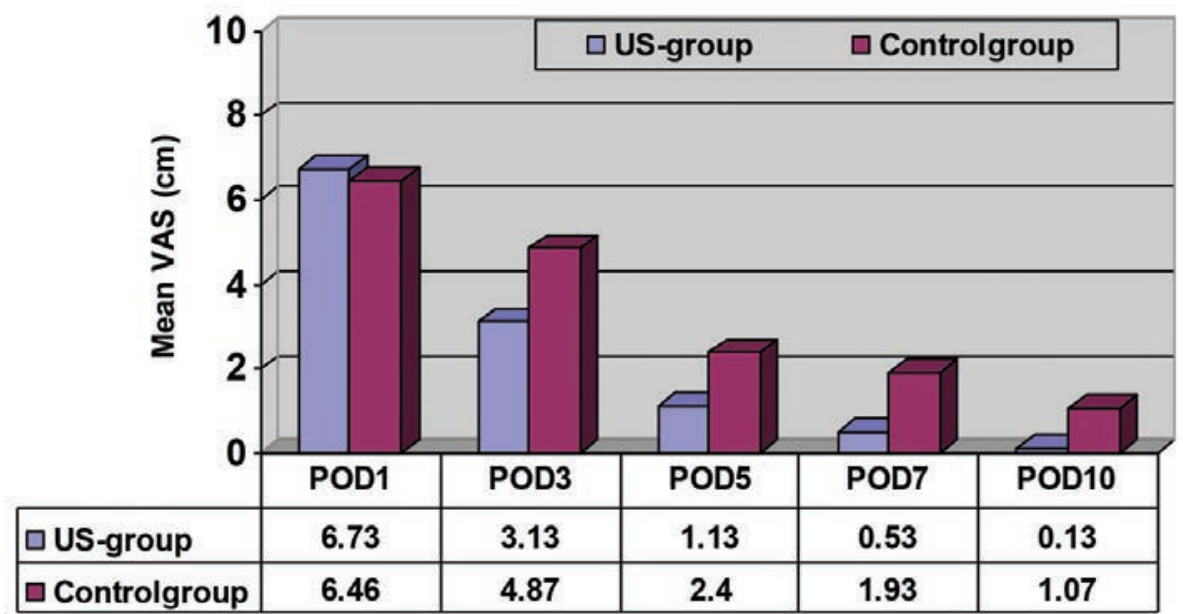

Figure (3): Average pain scores in visual analogue. 


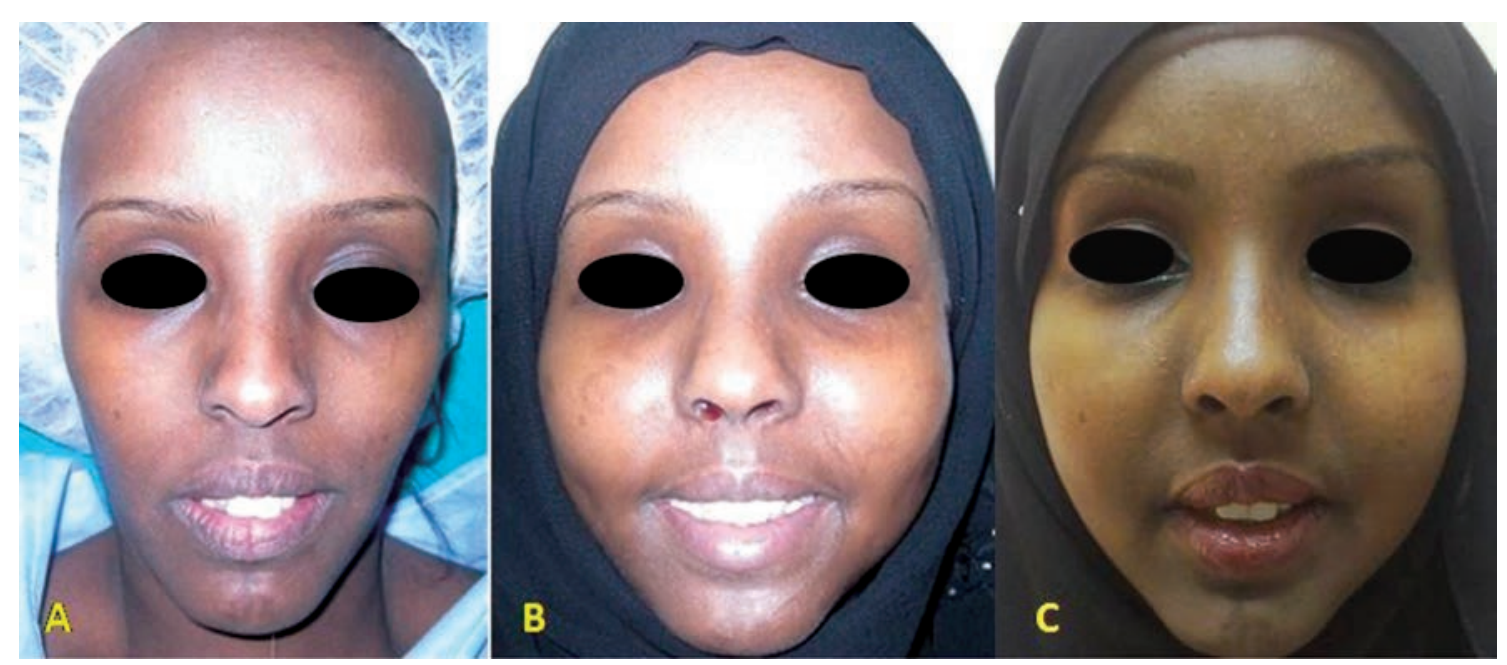

Figure (4): A. 35-year old female patient with thin face. B. 2 days after facial contouring by fat transfer. $C .7$ days post operative with marked reduction of facial edema and ecchymosis.

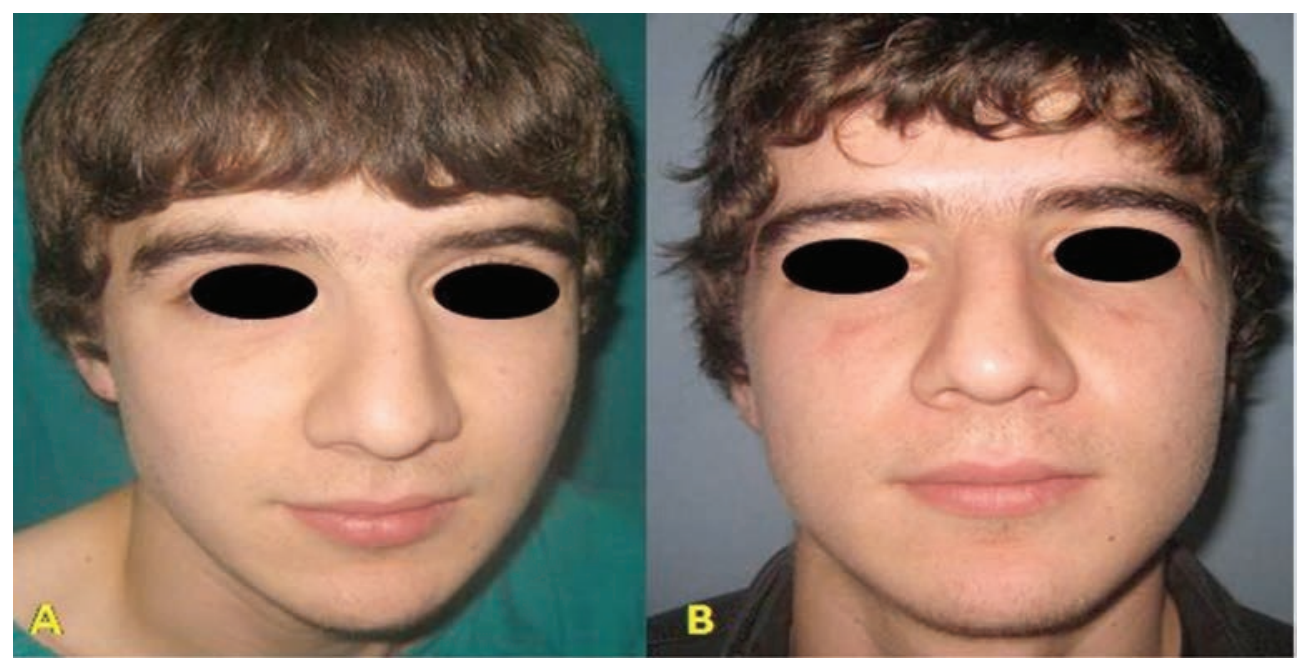

Figure (5):. A. 20-year old male patient with posttraumatic crooked nose. B. 2 weeks postoperative with residual ecchymosis of the face.

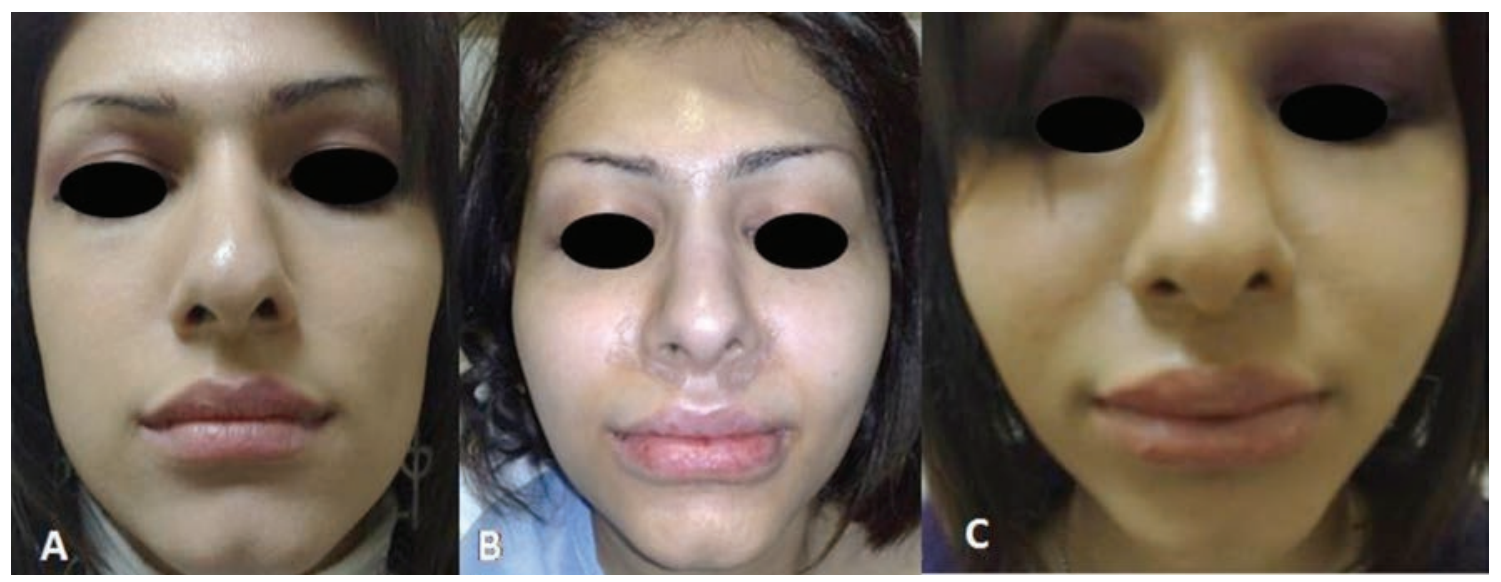

Figure (5): A. 26-year old female patient with slim face. B. 2 days after fat injection into cheeks and lips, with profound edema. $C .7$ days postoperative with the use of ultrasound treatment showing minimal edema. 
Table (1): Patients' characteristics.

\begin{tabular}{|l|c|c|c|c|c|}
\hline Variables & $\begin{array}{c}\text { US group } \\
(\mathbf{n = 1 5})\end{array}$ & $\begin{array}{c}\text { Control group } \\
(\mathbf{n = 1 5})\end{array}$ & $\begin{array}{c}\text { Total } \\
(\mathbf{n = 3 0})\end{array}$ & $\begin{array}{c}\text { Total } \\
(\mathbf{n = 3 0})\end{array}$ & P-value \\
\hline Age (years) & $41 \pm 5.33$ & $37 \pm 8.56$ & $39 \pm 6.56$ & $39 \pm 6.56$ & $0.9^{*}$ \\
\hline Sex N (\%) & & & & & \\
Male & $3(20 \%)$ & $4(26.7 \%)$ & $7(23.3 \%)$ & $7(23.3 \%)$ & $0.62^{*}$ \\
Females & $12(80 \%)$ & $11(73.3 \%)$ & $23(76.7 \%)$ & $23(76.7 \%)$ & \\
\hline Duration of Surgery (minutes) & $169.8 \pm 5.6$ & $170.3 \pm 9.0$ & $170.3 \pm 9.0$ & $170.3 \pm 9.0$ & $0.8^{*}$ \\
\hline Surgical Procedures N(\%) & & & & & \\
\hline Rhinoplasty & $6(40 \%)$ & $5(33.3 \%)$ & $11(36.7 \%)$ & $11(36.7 \%)$ & $0.46^{*}$ \\
Fat injection & $6(40 \%)$ & $6(40 \%)$ & $12(40 \%)$ & $12(40 \%)$ & $0.45^{*}$ \\
Blepharoplasty & $3(20 \%)$ & $4(26.7 \%)$ & $7(23.3 \%)$ & $7(23.3 \%)$ & $0.62^{*}$ \\
\hline
\end{tabular}

US; ultrasound, VAS =visual analogue scale, $*(p>0.05)$ non significant.

moderate pain. The mean pain scores revealed a statistical significant $(\mathrm{P}<0.05)$ reduction at PODs $5(1.13+$ versus2.4+), POD7 (0.53+ versus $1.93+)$ and POD10 $(0.13+$ versus $1.07+$ ) between US and control groups respectively.

\section{Discussion:}

This study examined the results of ultrasound treatment in patients who had facial aesthetic surgery confirmed by clinical examination of ecchymosis, edema and pain. There were significantly greater changes in all parameters for the ultrasound treatment group compared to control group. We reported that the maximum response to ultrasound treatment was between POD5 to POD10. This effect was seen in more than $95 \%$ of treated patients, with no recorded side effects. Therefore, ultrasound appears to be a safe and effective modality for enhancement of early recovery after facial aesthetic surgery when compared to control treatment.

It appears that exposure to continuous US during the initial 'inflammatory' phase of tissue repair causes heat, which would increase blood flow and edema in this area. ${ }^{31}$ In contrary, pulsed mode US causes the non-thermal effect on the tissue without measurable temperature changes. These can lead to an acceleration of this phase and reduction of the extent of tissue damage. ${ }^{9}$

The role of non-thermal mechanisms of US in tissue regeneration ${ }^{13}$ and repair ${ }^{14-17}$ has also been widely established. At a cellularlevel, it has been hypothesized that there are changes in diffusion rates and membrane permeability to ions ${ }^{18-20}$ due to acoustic streaming and stable cavitation. These can stimulate cell activity by up-regulation of signaling molecules, with associated reduction of edema formation. ${ }^{5,18-21}$ Moreover, during the inflammatory phase of the healing process, US can activate immune cells to migrate to the site of injury. Fyfe et al. showed induction of mast cell degranulation and histamine release in injury models in vivo using pulsed mode US of $0.5 \mathrm{~W} /$ $\mathrm{cm}^{2} .{ }^{22,23}$ Similar results were reported for dermal mast cells, demonstrating that US can accelerate the inflammatory healing phase for skin lesion/ulcers in vivo (Wistar rats, 0.75$\left.3 \mathrm{MHz}, 0.25-3 \mathrm{~W} / \mathrm{cm}^{2}\right) .{ }^{24}$ In related study, Young et $\mathrm{al}^{25}$ showed that ultrasound $(3 \mathrm{MHz}$, $0.5 \mathrm{~W} / \mathrm{cm}^{2}$ ) could stimulate macrophages in vitro to release fibroblast mitogenic factors, resulting in enhanced fibroblast proliferation.

By increasing the activity of these cells, the overall influence of therapeutic US is certainly pro-inflammatory rather than antiinflammatory. Studies which have tried to demonstrate the anti inflammatory effect of 
ultrasound have failed to do so (e.g.Hashish 1986, 1988), and have suggested that US is ineffective anti-inflammatory tool.

The pain relief following US therapy may be due to changes in pain perception mediated by circulating opiates. Exposure to US application enhances the release of substance Pfrom the nerve fibers, which induce analgesia and increased pain threshold by desensitizing the nociceptors. ${ }^{29}$ Furthermore, animal study demonstrated that US can reduce the sodiumpotassium ATPase pump activity, which if occurred in the neuronal plasma membrane, might inhibit the transduction of noxious stimuli and subsequent neural transmission, which may account in part for pain relief, which is often experienced following clinical exposure to therapeutic US .

The results of the current study are in constant with the findings of Rubin et al. ${ }^{12}$ who reported significant reduction in facial swelling and bruising after application of US in patients undergoing surgical cosmetic procedures. These results are supported by Van der Windt et al. ${ }^{5}$ who stated that ultrasound is currently used in physical therapy to reduce swelling, improve immobility and treat joint injuries.

Hashish et al., in his double blind study revealed that, US $\left(0.1-1.5 \mathrm{~W} / \mathrm{cm}^{2}\right)$ induced reduction in facial swelling and pain after oral surgery. ${ }^{30}$ Recently, Berna-Serna et al., reported significant reduction of rectus sheet hematoma, with associated relief of pain after US application $\left(1 \mathrm{MHz}, 1.5 \mathrm{~W} / \mathrm{cm}^{2}\right)$ for 8 to 12 minutes. ${ }^{31}$ The difference between this study and our study is in regards to the frequency of US (1MHz versus 3MHS) due to deep and superficial location of hematoma between the two studies.

Our study has several limitations. Because it was designed to assess both safety and efficacy, and because, to our knowledge, it was the first ultrasound study on live patients who were treated immediately after facial aesthetic surgeries. We used modest treatment parameters (intensity, pulsing and time) to make the benefit of US as efficient as possible to earliest repair phase, and thus have a promotional effect on the whole healing cascade. However, it is possible and likely that efficacy was not optimized. Therefore, more optimized exposure parameters (eg, continuous mode of US higher intensity, $1 \mathrm{MHz}$ ) may have induced greater effects. The small number of patients does not allow any definitive statement to be made regarding the efficacy of US therapy in accelerating recovery in patients following facial aesthetic surgeries, and the statistical power of the study is low. However, all subjects were treated in exactly the same way and at the same parameters.

In conclusion, we reported the safety and efficacy of the therapeutic US in expediting resolution of ecchymosis, edema and reduction of postoperative pain following facial aesthetic surgeries. The results of the study would encourage plastic surgeons and therapists to use the US as a valuable postoperative tool to accelerate resolution of ecchymosis, edema, and pain.

\section{References:}

1- DeFatta, RJ, Krishna S, Williams EF: Pulsed-Dye Laser for treating ecchymosis after facial cosmetic procedures. Arch Facial Plast Surg 2009; 11(2): 99-103.

2-Rubin A: Treating of postoperative bruisiing and edema with external ultrasound and manual lymphatic drainage. Plas Reconstr Surg 2002; 109: 1469-1471.

3-Shah SGS, Farrow A, Esnouf A: Availability and use of electrotherapy devices: A survey. International Journal of Therapy and Rehabilitation 2007; 14(6): 260-264.

4- Warden SJ, McMeeken JM: Ultrasound usage and dosage in sports physiotherapy. Ultrasound in Medicine and Biology 2002; 28(8): 1075-1080.

5- Dyson M: Mechanisms involved in therapeutic ultrasound. Physiotherapy 1987; 73: 116-120.

6- Kitchen SS, Partridge CJ: A review of therapeutic ultrasound. Physiotherapy 1990; 76: 593-600.

7- Hadjiargyrou M, McLeod K, Ryaby JP, Rubin C: Enhancement of fracture healing by low intensity ultrasound. Clin 
Orthop 1998; 355(suppl): 216-229.

8- Van der Windt DA, van der Heijden GJ, van der Berg SG, et al: Ultrasound therapy for musculoskeletal disorders: A systematic review. Pain 1999; 81: 257-271.

9- Speed CA: Therapeutic ultrasound in soft tissue lesions. Rheumatology 2001; 40: 1331-1336.

10-Baker KG, Robertson VJ, Duck FA: A review of therapeutic ultrasound: Biophysical effects. Phys Ther 2001; 81: 1351-1358.

11-Van der Heijden GJ, van der Windt DA, Van der Winter AF: Physiotherapy for patients with soft tissue shoulder disorders: A systematic review of randomized trials. BMJ 1997; 315: 25-30.

12-Robertson VJ, Baker KG: A review of therapeutic ultrasound: Effectiveness studies. Phys Ther 2001; 81: 1339-1350.

10-Dixon JS, Bird HA: Reproducibility along a $10 \mathrm{~cm}$ vertical visual analogue scale. Ann Rheum Dis 1981; 40: 87-89.

11-Sabagh RM, Ali A, Taher MO: Role of ultrasound in minimizing the postoperative swelling and pain after cosmetic facial surgery. Sc J Az Med Fac(Girls) 2006; 27(2): 1877-1886.

12-Piso DU, Eckardt A, Schafer P, Gehrke A: Early rehabilitation of head-neck edema after curative surgery for orofacial tumors. Am J Phys Med Rehabil 2001; 80: 261-269.

13-Dyson M, Pond JB, Joseph J, et al: The stimulation of tissue regeneration by means of ultrasound. Clin Sci1 968; 32(2): 273-285.

14-Dyson M, Franks C, Suckling J: Stimulation of healing of varicose ulcers by ultrasound. Ultrasonic 1976; 14(5): 232-236.

15-Paul BJ, Lafrotto CW, Dawson AR, et al: Use of ultrasound in treatment of pressure sores in patients with spinal cord injury. Arch Phys Med Rehabil 1969; 41: 438-440.

16-Webster DE, Pond JB, Dyson M, Harvey $\mathrm{W}$ : The role of cavitation in the in vitro stimulation of protein synthesis in human fibroblasts by ultrasound. Ultrasound
Med Biol 1978; 4(4): 343-351.

17-Webster DE, Harvey W, Dyson M, Pond JB: The role of ultrasound- induced cavitation in the 'in vitro' stimulation of collagen synthesis in human fibroblasts. Ultrasonics 1980; 18: 33-37.

18-Dinnot M, Young S, Crumt A: The significance of membrane changes in the safe and effective use of therapeutic and diagnostic ultrasound. Phys Med Biol 1989; 34(11): 1543-1552.

19-Mortimer AJ, Dyson M: The effect of therapeutic ultrasound on calcium uptake in fibroblasts. Ultrasound Med Biol 1988; 14(6): 499-506.

20-Ryaby J, et al: Low intensity pulsed ultrasoundincreasescalciumincorporation in both differentiating cartilage and bone cell cultures. Trans Orthop Res Soc 1989; 14:

21-Dyson M: Therapeutic applications of ultrasound. In: Biological effects of ultrasound. Churchill Livingstone (Publisher); 1985; p.135-155.

22-Fyfe M, Chahl L: Mast cell degranulation: a possible mechanism of action of therapeutic ultrasound. Ultrasound Med Biol 1982; 8(Suppl): 62-65.

23-Fyfe M, Chahl L: Mast cell degranulation and increased vascular permeability induced by 'therapeutic' ultrasound in the rat ankle joint. Br J Exp Pathol 1984; 65 (6): 671-676.

24-Dyson M, Luke D: Induction of mast cell degranulation in skin by ultrasound, ultrasonics, ferroelectrics and frequency control. IEEE Transactions 1986; 33(2): 194-201.

25-Young S, Dyson M: Macrophage responsiveness to therapeutic ultrasound. Ultrasound Med Biol 1990; 16(8): 809-816.

26-Devcic-Kuhar B, Pfaffenberger S, Groschl $\mathrm{M}$, et al: In vitro thrombolysis enhanced by standing and travelling ultrasound wave fields. Ultrasound Med Biol 2002; 28: 1181-1187.

27-Francis CW, Onundarson PT, Carstensen EL, et al: Enhancement of fibrinolysis in vitro by ultrasound. J Clin Invest 1992; 
90: 2063-2068.

28-Harpaz D, Chen X, Francis CW, et al: Ultrasound enhancement of thrombolysis and reperfusion in vitro. $J$ Am Coll Cardiol 1993; 21: 1507-1511.

29-Hashish I, Hai HK, Harvey W, Feinmann C, Harris M: Reduction of postoperative pain, swelling by ultrasound treatment: A placebo effect. Pain 1988; 33: 303-311.
30-Hashish I, Hai HK, Harvey W, Harris M: Anti-inflammatory effects of ultrasound therapy: Evidence for major placebo effect. Br J Rh 1986; 77-81.

31-Berna'-Serna JD, Sa'nchez-Garre J, Madrigal M, et al: Ultrasound therapy in rectus sheath hematoma. Phys Ther 2005; 85: 352-357. 